ARAŞTIRMA / RESEARCH

\title{
An age-related investigation of audio-vestibular symptoms in individuals with Covid-19 positive
}

Covid-19 pozitif bireylerde yaşla ilgili odyo-vestibüler belirtilerin oranı

\author{
Bünyamin Çıldır
}

${ }^{1}$ Ankara Yıldırım Beyazit University Health Sciences Faculty, Language and Speech Therapy Department, Ankara, Turkey

\begin{abstract}
Purpose: The aim of this study was to determine the rate of audio-vestibular complaints by COVID-19 patients during the disease period and to evaluate the symptoms they encountered during the disease process in accordance with age and gender.
\end{abstract}

Materials and Methods: This study was conducted with a total of 1437 patients $(738 \mathrm{M}, 699 \mathrm{~F})$ with a positive PCR test, aged 18-80 years, between 15 June 2020 and 10 February 2021. The patients were divided into five groups according to age. 1437 patients were questioned about their complaints on the second day of the disease, but only 656 of 1437 patients were questioned on the fourteenth day of the disease. Finally, 1437 patients were called again for the third time in the third month of the disease, but only 340 of these patients could be reached.

Results: Of the 1437 patients, 524 (36.5\%) had more frequent audio-vestibular complaints than common complaints. During the 14-day disease period, dizziness or vertigo $(3.4 \%$ to $19.4 \%)$, tinnitus $(1.02 \%$ to $19.4 \%)$, pressure in the ears $(1.1 \%$ to $5.6 \%)$, difficulty understanding speech in noise $(0.13 \%$ to $26 \%)$, decreased sound tolerance $(0.13 \%$ to $6.4 \%)$, speech difficulties $(0.13 \%$ to $5 \%)$, and earache $(1.4 \%$ to $7.8 \%)$ reportedly increased compared with the first day of the disease. Fiftytwo $(15.3 \%)$ of 340 patients reported that they lost their sense of taste, $90(26.4 \%)$ lost their sense of smell, and 14 $(4.11 \%)$ reported intermittent vertigo in the third month of COVID-19 infection.

Conclusion: Symptoms such as dizziness, tinnitus, hoarseness, swallowing difficulty, fever, and decreased sound tolerance can often be seen in the later days of COVID-19 disease.

Keywords:. Tinnitus; vertigo; coronavirus; COVID-19; speech and swallowing disorder

\section{Öz}

Amaç: Bu çalışmanın amac1, COVID-19 tanısı alan hastaların hastalık döneminde karșılaștıkları odyovestibüler şikayet oranlarını belirlemek ve bu hastaların yaş ve cinsiyet özelliklerine göre hastalı sürecinde karşılaştıkları semptomları değerlendirmektir.

Gereç ve Yöntem: Bu çalışma, 15 Haziran 2020 ile 10 Şubat 2021 tarihleri arasında, 18-80 yaşları arasında, PCR testi pozitif olan toplam 1437 hasta (738 E, $699 \mathrm{~K}$ ) ile gerçekleştirildi. Hastalar yaşlarına göre beş gruba ayrıldı. 1437 hastanın șikayetleri hastalığın 2. gününde sorgulanırken, 1437 hastanın sadece 656'sı hastalığın 14. gününde ulaşıldı ve sorgulandı. Son olarak, hastalığın üçüncü ayında 1437 hasta üçüncü kez tekrar aranmış ancak bu hastalardan da sadece 340 'Ina ulaşılabilmiştir.

Bulgular: 1437 hastanın 524 'ünde (\% 36.5) yaygın şikayetlerden daha sık odyo-vestibüler şikayetler vardı. 14 günlük hastalık sürecinde, baş dönmesi veya vertigo (\%3.4 - \%19.4), kulak çınlaması (\%1.02 -\%19.4), kulaklarda basınç (\%1.1 -\%5.6), gürültüyü anlamada zorluk (\%0.13 $\% 26)$, konuşmada güçlük $(\% 0.13-\% 5)$ ve kulak ağrisı (\%1.4 - \%7.8) semptomlarında artış bildirilmiştir. Baş dönmesi 60 yaş üstü bireylerde daha yaygındı. Kovid-19 hastalığının üçüncü ayında, 340 hastanın 52'si (\%15.3) tat alma alamadığını, 90'1 (\%26.4) koku alamadığını ve 14'ü (\%4.11) ise aralıklı olarak baş dönmesi veya dengesizlik şikayetinin olduğunu bildirdi.

Sonuç: Koronavirüs hastalığının ilerleyen günlerinde baş dönmesi, kulak çınlaması, ses kısıklı̆̆ı, yutma güçlüğü, ateș ve azalan ses töleransı gibi belirtiler sıklıkla görülebilmektedir.

Anahtar kelimeler: Tinnitus; baş dönmesi; koronavirüs; COVID -19; konuşma ve yutma bozukluğu 


\section{INTRODUCTION}

Coronavirus disease (COVID-19), which first appeared in Wuhan, China, and spread rapidly worldwide in a short time, causing severe acute respiratory syndrome, pneumonia, and respiratory failure, was declared a pandemic by the World Health Organization $(\mathrm{WHO})^{1-4}$. The disease is caused by the new coronavirus SARS-CoV-2, which resembles the severe acute respiratory syndrome (SARS-CoV) that emerged in $2003^{5}$. As of January 2021, more than 92 million cases were diagnosed, and 2.06 million patients reportedly died due to coronavirus worldwide (involving more than 177 countries) ${ }^{6}$. The first case in Turkey was reported on 13 March 2020.

The COVID-19 pandemic is observed on a spectrum, from clinically mild symptoms, such as cold, to severe acute respiratory syndrome ${ }^{5}$. Although the source of the COVID-19 pandemic is not precisely known, the rate of contagiousness of this pandemic from person to person is high and can cause respiratory infections similar to the common cold in patients, as well as deadly, such as MERS and SARS endemics ${ }^{1}$. Fever, cough, sore throat, headache, muscle pain, diarrhea, dyspnea, loss of sense of smell, and taste are the most common symptoms in the COVID-19 pandemic p $^{3,7,8}$. At the same time, it has been stated that the COVID-19 pandemic is associated with central and peripheral nervous system symptoms, such as cerebrovascular diseases and visual impairment, as well as many otolaryngological symptoms according to age and gender ${ }^{9,10}$.

The aim of this study was to determine the rate of audio-vestibular complaints by COVID-19 patients during the disease period and to evaluate the symptoms they encountered during the disease period in accordance with age and gender. The findings of the study will provide useful information to the literature on otolaryngologist and audiologist applications for the diagnosis and treatment of COVID-19 cases.

\section{MATERIALS AND METHODS}

This study was approved by the Ankara Yıldırım Beyazit University Ethics Committee (Approve no: 2020/58), the Ministry of Health of the Republic Ethics Committee of Turkey (Approve no: 2020-0716T22_16_27), and the Ethics Committee of the General Directorate of Public Health. Informed consent forms were sent to the patients via e-mail, which they signed.

\section{Participants}

Patients who were admitted to the hospitals affiliated with the Ministry of Health in Ankara Çankaya district between 15 July 2020 and 10 January 2021 and who were confirmed positive for COVID-19 were included in the study. All the patients who participated in our preliminary study were called by a physician working on the COVID-19 filiation team at the Provincial Health Directorate of Ankara, Turkey. A total of 3617 adults aged 18-80 years who had positive SARS-CoV-2 test results participated in the study. All the patients who participated in our preliminary study were called by a physician working on the COVID-19 filiation team at the Provincial Health Directorate of Ankara, Turkey.

A total of 3617 adults aged 18-80 years who had positive SARS-CoV-2 test results participated in the study. All patients' diagnoses were confirmed simultaneously using real-time polymerase chain reaction (RT-PCR) testing of their nasopharyngeal and oropharyngeal swab specimens. All patient information about the COVID-19 pandemic was obtained from the patients with their own consent. Enlightened consent forms were sent to all patients by e-mail and were signed by the patients.

Patients were first asked about their medical history, including their comorbidities and medications, by a native Turkish physician. An information form about COVID-19 audio-vestibular symptoms was created by one audiologists and a physician, and the questions in this information form were asked to the patients.

We prepared a COVID-19 patient history form to record general symptoms, audio-vestibular symptoms of COVID-19, and other symptoms, such as speech, swallowing, and voice disorders. According to the literature, the most common general symptoms reported were fever, cough, fatigue, arthralgia, and dyspnea 11,12. Some of the COVID-19 patient history form items were earache, loss of hearing, difficulty understanding speech in noise, difficulty swallowing, speech disorder, voice disorder, and vertigo. The information form (COVID-19 patient history form) was read and recorded item-by-item by a physician in the filiation team (Appendix 1). All questions and general symptoms in the patient story form were again asked by the same doctor, posing queries about ear surgery, ear fullness, common dizziness, tinnitus, or buzzing 
in the ear before the COVID-19 pandemic. In addition to general health, such as headache, fever, vomiting, nausea, diarrhea, and dyspnea, the index addressed audio-vestibular symptoms and speech, voice, and swallowing disorders.

One day after the diagnosis of the disease, a total of 3617 patients were interviewed by phone and asked about their general conditions and symptoms. Patients who did not meet the inclusion criteria were excluded from the study, and 1437 of the total sample of patients were included in the study. Each of the 1437 patients were asked by phone about their general conditions and symptoms. Further, these 1437 patients were called for a second time on the thirteenth or fourteenth day of the disease, counted from the day of the positive RT-PCR result. Only 656 of 1437 patients could be reached on the thirteenth or fourteenth day of the disease. Finally, all 1437 patients were called for a third time after three months to inquire about the long-term findings from the COVID-19 pandemic. Only 340 of 1437 patients could be reached in the third month of the disease.

All patients participating in our study consisted of patients who were quarantined at home after a diagnosis of COVID-19. Patients with any vestibular disorder, neurological disorder, ear surgery, frequent voice disorder, shortness of breath, or tinnitus in the pre-COVID-19 period were not included in the study.

The patients were divided into five groups according to age (18-30 years for Group I, 30-40 years for Group II, 40-50 years for Group III, 50-60 years for Group IV, and $>60$ years for Group V). Patients were asked about their complaints and medical history during the disease period after being diagnosed with COVID-19.

\section{Measures}

\section{COVID-19 patient history form}

The patient story form is an information form created to report patients' general complaints and audiovestibular complaints during the COVID-19 disease period and before the COVID-19 disease. The information form consists of three subsections and 26 questions. The first part covers general complaints, such as cough and diarrhea, during the COVID-19 disease period, the second part contains questions about the quality of life of the patients, and the third part contains questions about the audiovestibular complaints of the patients.

\section{Audio-Vestibular Symptom Questionnaire}

The audio vestibular symptoms within the questionnaire were included after reviewing clinical studies of the literature that reported otolaryngological symptom prevalence in COVID-19 patients. Most of the questions on the audiovestibular symptom questionnaire that make up this information form were selected from the spatial hearing questionnaires and speech spatial and qualities of hearing scale questionnaires, the validity and reliability of which were verified by Ç1ldir et al. ${ }^{13,14}$, the hearing impairment scales, the validity and reliability of which were verified by Aksoy et al..$^{15}$, and the vestibular disorders activities of daily life questionnaires, the validity and reliability of which were verified by Çinar et al. ${ }^{16}$. At the same time, questions containing middle ear infection information were selected from the chronic otitis media questionnaire conducted by Doruk et al. ${ }^{17}$.

After preparing the patient history form, a preliminary study was performed for the audiovestibular symptom questionnaire. This preliminary study was conducted on 133 patients aged $18-50$ years (mean age $38 \pm 14.2$ years) whose RT-PCR and oropharyngeal swab specimens were positive, and test-retest reliability was checked.

Internal consistency was evaluated with the coefficient Cronbach's Alpha. The Cronbach's Alpha value of the audio-vestibular symptom questionnaire was found to be 75.1 for those patients. The Cronbach's Alpha value of the audio-vestibular symptom questionnaire was calculated, and this value was found to be above the critical value of 0.7 , which is the questionnaire usability level. The high $\alpha$ values indicate that the internal consistency reliability of the questionnaire is good. The item-total correlation for these patients was between 0.45 and 0.98 . The testretest correlation was sufficient $(r=0.614, p=0.027)$. A doctor in charge of the filiation read all the questions in the patient story form to the patients in clear language, and the information received from the patients was recorded.

\section{Statistical analysis}

Data analysis was performed using SPSS v.22.0 (Statistical Package for Social Sciences) (SPSS Inc., Chicago, IL, US). Statistics for all data were presented as mean \pm standard deviation, number, and percentages. Pearson chi square tests were used in comparisons of categorical data. Symptom 
differences between the genders and ages of the five groups during the COVID-19 disease period were compared using the one-way ANOVA with post-hoc test. Cronbach's alpha and item-total correlation internal consistency was performed for factor analysis of the audio-vestibular symptom index. Total sample size was calculated with respect to the chisquare test performed for the difference between age groups considering the any of audio-vestibular symptoms. Sample size was obtained as 133 participants for 4 degree of freedom, a type 1 error of 0.05 , a type 2 error of 0.20 , and the medium effect size of $\mathrm{w}=0.30$, which is suggested by Cohen, by using pwr package in RStudio Software with $\mathrm{R}$ language v. $4.1^{18-20}$. For all parameters, $p \leq 0.05$ was considered statistically significant.

\section{RESULTS}

The mean age of all 1437 patients was $40.6 \pm 14.3$ years. Of the participants, $738(51.4 \%)$ were male and $699(48.6 \%)$ were female. Most of these patients $(87 \%)$ stated that they had protected themselves against the virus with extreme care. A total of 297 $(20.7 \%)$ patients stated that they went to the hospital when they had mild flu symptoms (such as runny nose, weakness), and the 2019-nCoV test results were positive (see Table 1). All patients were quarantined at home.

When all 1437 patients were called on the second day after their diagnosis of COVID-19, the following number of patients reported the following symptoms: $49(3.4 \%)$ dizziness or vertigo; 358 $(24.9 \%)$ cough; 395 (27.4\%) fatigue; 69 (4.8\%) dyspnea; $38(2.6 \%)$ chill; $14(0.9 \%)$ tinnitus; 129 $(16.5 \%)$ arthralgia and notalgia; 194 (24.8\%) headache; $2(0.13 \%)$ difficulty understanding speech in noise; $2(0.13 \%)$ decreased sound tolerance; 2 $(0.13 \%)$ speech disorder; $32(2.2 \%)$ dysphagia; 66 (4.6\%) aphonia; $284(19.7 \%)$ ageusia; 292 (20.3\%) anosmia; $102(7 \%)$ sore throat; $20(1.4 \%)$ otalgia; 16 $(1.1 \%)$ pressure in the ear; $58(4.03 \%)$ diarrhea; 81 $(5.6 \%)$ fever; and 31 (2.15) nasal congestion (see Table 2 and Figure 1).

The comorbidities of the patients were as follows: 1 $(0.06 \%)$ anemia; 64 (4.45\%) hypertension; 26 (1.8\%) asthma; $19(1.32 \%)$ cardiovascular diseases; and 9 $(0.6 \%)$ hypothyroid cases. Other comorbid diseases were observed less frequently (see Table 1).

On the second day of the disease, four patients exhibited symptoms such as sore throat, ear discharge, pressure in the ear, nasal congestion, fever, and/or tinnitus. The average age of these patients was $43.7 \pm 8$ years. In addition, sore throat, ear discharge, pressure in the ear, fatigue, and/or vertigo symptoms were reported by 11 patients. The average age of these patients was $46.8 \pm 10.7$ years. Two patients reported both a comorbidity (hypertension or cardiovascular diseases) and tinnitus, vertigo, and/or other audio-vestibular complaints. On the second day of the disease, tinnitus was reported by only two of the patients who were over 50 years old, and balance complaints were reported by 15 patients. No one over the age of 30 had tinnitus or difficulty understanding speech in noise. There were no statistically significant differences in the data with regard to the genders and ages of the five groups on the second day after their diagnosis of COVID-19 $(\chi 2$ $=1.396, \mathrm{sd}=4, \mathrm{p}>0.845)$. Loss of taste and smell was reported less frequently in the 50-60 and $>60$ age groups than in the other groups. The incidence of fever was lower in individuals over 60 years of age than in other individuals.

The rate of complaining about fatigue, headache, difficulty understanding speech in noise, and decreased sound tolerance was higher in women than in men. Weakness, pain in the joints, headache, difficulty understanding in noise, decreased sound tolerance, difficulty speaking, hoarseness, loss of taste and smell, sore throat, earache, pressure in the ear, sensitivity to sound and light, and nasal congestion were more common in men, whereas more women reported fever (see Table 3). Three patients had conjunctivitis.

Of the other 656 patients who were called fourteen days after the diagnosis of the disease, the following number of patients reported the following symptoms: $127(19.4 \%)$ dizziness or vertigo; 73 (11.1\%) cough; 79 (12\%) fatigue; 87 (13.3\%) nausea and vomiting; $116(17.7 \%)$ dyspnea; 120 (18.3\%) chill; 127 (19.4\%) tinnitus; 202 (30.8\%) arthralgia and notalgia; $313(47.7 \%)$ headache; 173 (26.4\%) difficulty understanding speech in noise; 42 (6.4\%) decreased sound tolerance; $33(5 \%)$ speech disorder; $45(6.9 \%)$ dysphagia; 110 (16.8\%) aphonia; 219 (33.4\%) ageusia; $362(55.2 \%)$ anosmia; 238 (36.3\%) sore throat; $51(7.8 \%)$ otalgia; $37(5.6 \%)$ pressure in the ear; $113(17.2 \%)$ diarrhea; 130 (19.8\%) fever; and $4(0.6 \%)$ nasal congestion (see Table 2$)$.

On the fourteenth day of the disease, 38 patients exhibited symptoms such as sore throat, ear discharge, pressure in the ear, and/or nasal 
congestion, as well as tinnitus. In addition, sore throat, ear discharge, pressure in the ear, fatigue, and/or vertigo symptoms were observed in 13 individuals. Seventeen patients reported both a comorbidity and tinnitus, vertigo, and/or other audiovestibular complaints.

On the fourteenth day of the disease, tinnitus was reported in 53 of the patients who were over 50 years old, and balance complaints were reported in 40 patients. Meanwhile, 24 patients had difficulty understanding speech in noise; they also experienced tinnitus. In particular, an increase in symptoms such as dizziness or vertigo (3.4\% to $19.4 \%$ ), tinnitus $(1.02 \%$ to $19.4 \%)$, pressure in the ears $(1.1 \%$ to $5.6 \%)$, shortness of breath (4.8\% to $17.7 \%$ ), arthralgia and notalgia $(16.5 \%$ to $30.8 \%)$, headache $(24.8 \%$ to $47.7 \%)$, difficulty understanding speech in noise $(0.13 \%$ to $26.4 \%)$, decreased sound tolerance $(0.13 \%$ to $6.4 \%)$, speech disorder $(0.13 \%$ to $5 \%)$, dysphagia $(2.2 \%$ to $6.9 \%)$, aphonia $(4.6 \%$ to $16.8 \%)$, ageusia $(19.7 \%$ to $33.4 \%)$, anosmia $(20.3 \%$ to $55.2 \%)$, sore throat $(7 \%$ to $36.3 \%)$, and otalgia $(1.4 \%$ to $7.8 \%)$ over time was observed during 14 days of the pandemic.

Regarding the comorbidities of the 656 patients, we observed $1(0.15 \%)$ anemia, $42(6.4 \%)$ hypertension, 17 asthma (2.6\%), $17(2.59 \%)$ cardiovascular diseases, and $1(0.1 \%)$ hypothyroid; other comorbid diseases were observed less frequently. Of the 656 patients, $307(46.8 \%)$ were male and 349 (53.2\%) were female. The average age of the group was 39.09 \pm 13.32 years (Table 1$)$. A statistical difference was observed only in terms of dizziness or vertigo $\left(\chi^{2}=\right.$ 15.478, $\mathrm{Sd}=4, \mathrm{p}=0.003)$, arthralgia $\left(\chi^{2}=15.259, \mathrm{Sd}\right.$ $=4, \mathrm{p}=0.022)$, and notalgia $\left(\chi^{2}=1.583, \mathrm{Sd}=4, \mathrm{p}>\right.$ $0.003)$ between the age of 60 and over and other age groups. Dizziness was more common in individuals over 60 years of age (47 patients). A statistical difference in headache was observed between the 50 60 years old group and the $40-50$ years old group $\left(\chi^{2}=\right.$ $10.829, \mathrm{Sd}=4, \mathrm{p}=0.049)$. Difficulty understanding in noise, decreased sound tolerance, and speech disorder were found to be more common in the group over 60 years of age compared to other groups.

There was a statistically significant difference in swallowing difficulty in the 30-40-year-old group and in the over 60-year-old group compared to the other groups $\left(\chi^{2}=6.789, \mathrm{Sd}=4, \mathrm{p}=0.037\right)$. A statistically significant difference was found between all groups for loss of smell $\left(\chi^{2}=19.958, \mathrm{Sd}=4, \mathrm{p}=0.001\right)$.

Sensitivity to sound and light and nasal congestion were observed more in the individuals over 60 years old than in the other age groups. Most of the disease symptoms were observed more in women than men (Table 3). Arthralgia and notalgia, dizziness, vertigo, headache, difficulty understanding in noise, being distracted by noise, speech disorder, aphonia, loss of taste and smell, sore throat, ear pain, feeling of pressure in the ear, sensitivity to sound and light, and nasal congestion were more common in women, whereas fatigue, tinnitus, and swallowing difficulty were observed more in men (Table 3).

There was no statistical difference between the groups in terms of gender $(p=0.238)$. Compared to symptoms on the first two days after a positive COVID-19 diagnosis, there was an increase in the rate of fever $(5.6 \%$ to $19.8 \%)$, headache Arthralgia $(24.7 \%$ to $47.7 \%)$, and dyspnea $(4.8 \%$ to $17.1 \%)$ by the 14th day of the disease (see please Figure 1). There was a significant increase in the reporting of all symptoms other than audio-vestibular symptoms (except nasal obstruction) over time $(\mathrm{p}<0.05)$.

Table 1. Demographic characteristics of patients

\begin{tabular}{|l|c|c|c|}
\hline & \multicolumn{3}{|c|}{ The period of the disease } \\
\hline & $\begin{array}{c}\text { Patients } \\
\left(\mathbf{2}^{\text {th }} \mathbf{d a y}\right)\end{array}$ & $\begin{array}{c}\text { Patients } \\
\left.\mathbf{1}^{\text {th }} \mathbf{d a y}\right)\end{array}$ & $\begin{array}{c}\text { Patients } \\
\mathbf{3}^{\text {th }} \text { month) }\end{array}$ \\
\hline Number of patient (n) & 1437 & 656 & 340 \\
\hline Age (Mean士 Sd) & $40.6 \pm 14.3$ & $39.09 \pm 13.3$ & $40.1 \pm 13.5$ \\
\hline Sex (Female/Male) & $662 / 738$ & $349 / 307$ & $181 / 159$ \\
\hline Comorbid diseases [n/\%] & & & - \\
\hline Anemia & $1 / 0.06 \%$ & $1 / 0.15 \%$ & $22 / 6.4$ \\
\hline Hypertension & $64 / 4.5 \%$ & $42 / 6.4 \%$ & $14 / 4.1$ \\
\hline Asthma & $26 / 1.8 \%$ & $17 / 2.6 \%$ & $16 / 4.7$ \\
\hline Cardiovascular diseases & $19 / 1.3 \%$ & $17 / 2.6 \%$ & $7 / 2.1$ \\
\hline Hypothyroid cases & $9 / 0.6 \%$ & $1 / 0.1 \%$ & \\
\hline
\end{tabular}

Sd: Standard deviation 
Table 2. Patient symptom frequency of the disease in different age groups within 2nd day and during 14 days and 3 month of pandemics

\begin{tabular}{|c|c|c|c|c|c|c|c|c|c|c|c|c|c|c|c|c|c|c|}
\hline & \multirow{2}{*}{ 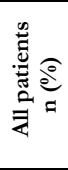 } & \multicolumn{5}{|c|}{ Age groups within the first 2 days } & \multirow{2}{*}{ 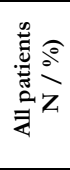 } & \multicolumn{5}{|c|}{ Age groups during 14 days } & \multirow{2}{*}{ 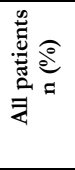 } & \multicolumn{5}{|c|}{ Age groups during 3 month } \\
\hline & & $\begin{array}{l}18-30 \\
\text { years }\end{array}$ & $\begin{array}{l}30-40 \\
\text { years }\end{array}$ & $\begin{array}{l}40-50 \\
\text { years }\end{array}$ & $\begin{array}{l}50-60 \\
\text { years }\end{array}$ & $\begin{array}{l}\text { Over } \\
60 \\
\text { years }\end{array}$ & & $\begin{array}{l}18-30 \\
\text { years }\end{array}$ & $\begin{array}{l}30-40 \\
\text { years }\end{array}$ & $\begin{array}{l}40-50 \\
\text { years }\end{array}$ & $\begin{array}{l}50-60 \\
\text { years }\end{array}$ & $\begin{array}{l}\text { Over } \\
60 \\
\text { years }\end{array}$ & & $\begin{array}{l}18-30 \\
\text { years }\end{array}$ & $\begin{array}{l}30-40 \\
\text { years }\end{array}$ & $\begin{array}{l}40-50 \\
\text { years }\end{array}$ & $\begin{array}{l}50-60 \\
\text { years }\end{array}$ & $\begin{array}{l}\text { Over } \\
60 \\
\text { years }\end{array}$ \\
\hline Patients & 1437 & $\begin{array}{l}338 \\
(23.5)\end{array}$ & $\begin{array}{l}367 \\
(25.5)\end{array}$ & $\begin{array}{l}302 \\
(21)\end{array}$ & $\begin{array}{l}240 \\
(16.7)\end{array}$ & $\begin{array}{l}190 \\
(13.2)\end{array}$ & 656 & $\begin{array}{l}183 \\
(27.9)\end{array}$ & $\begin{array}{l}180 \\
(27.4)\end{array}$ & $\begin{array}{l}136 \\
(20.7)\end{array}$ & $\begin{array}{l}110 \\
(16.8)\end{array}$ & $\begin{array}{l}47 \\
(7.2)\end{array}$ & 340 & $\begin{array}{l}55 \\
(27.9)\end{array}$ & $\begin{array}{l}94 \\
(27.6)\end{array}$ & $\begin{array}{l}61 \\
(17.9)\end{array}$ & $\begin{array}{l}79 \\
(23.2)\end{array}$ & $\begin{array}{l}11 \\
(3.2) \\
\end{array}$ \\
\hline Fatigue & $\begin{array}{l}395 \\
(27.4)\end{array}$ & $\begin{array}{l}106 \\
(3.4)\end{array}$ & $\begin{array}{l}84 \\
(22.9)\end{array}$ & $\begin{array}{l}84 \\
(27.8)\end{array}$ & $\begin{array}{l}79 \\
(32.9) \\
\end{array}$ & $\begin{array}{l}42 \\
(22.1)\end{array}$ & \begin{tabular}{|l|}
113 \\
$(17.2)$ \\
\end{tabular} & $\begin{array}{l}21 \\
(11.5)\end{array}$ & $\begin{array}{l}21 \\
(11.7)\end{array}$ & $\begin{array}{l}20 \\
(12.3)\end{array}$ & $\begin{array}{l}13 \\
(11.8)\end{array}$ & $\begin{array}{l}4 \\
(8.5)\end{array}$ & & & & & & \\
\hline Fever & $\begin{array}{l}81 \\
(5.6)\end{array}$ & $\begin{array}{l}12 \\
(3.6)\end{array}$ & $\begin{array}{l}24 \\
(6.5)\end{array}$ & $\begin{array}{l}18 \\
(6)\end{array}$ & $\begin{array}{l}23 \\
(9.6)\end{array}$ & $\begin{array}{l}4 \\
(2.1)\end{array}$ & $\begin{array}{l}130 \\
(19.8) \\
\end{array}$ & $\begin{array}{l}36 \\
(19.7)\end{array}$ & $\begin{array}{l}36 \\
(20)\end{array}$ & $\begin{array}{l}22 \\
(13.5)\end{array}$ & $\begin{array}{l}27 \\
(24.5)\end{array}$ & $\begin{array}{l}9 \\
(19.1)\end{array}$ & & & & & & \\
\hline Cough & $\begin{array}{l}358 \\
(24.9) \\
\end{array}$ & $\begin{array}{l}87 \\
(25.7) \\
\end{array}$ & $\begin{array}{l}89 \\
(24.3)\end{array}$ & $\begin{array}{l}78 \\
(25.8)\end{array}$ & $\begin{array}{l}67 \\
(27.9) \\
\end{array}$ & $\begin{array}{l}37 \\
(19.5)\end{array}$ & $\begin{array}{l}73 \\
(11.1)\end{array}$ & $\begin{array}{l}19 \\
(10.4)\end{array}$ & $\begin{array}{l}19 \\
(10.6)\end{array}$ & $\begin{array}{l}19 \\
(11.7)\end{array}$ & $\begin{array}{l}12 \\
(10.9)\end{array}$ & $\begin{array}{l}4 \\
(8.5) \\
\end{array}$ & & & & & & \\
\hline Dyspnea & $\begin{array}{l}69 \\
(4.8)\end{array}$ & $\begin{array}{l}30 \\
(8.9) \\
\end{array}$ & $\begin{array}{l}12 \\
(3.3) \\
\end{array}$ & $\begin{array}{l}16 \\
(5.3) \\
\end{array}$ & $\begin{array}{l}9 \\
(3.8) \\
\end{array}$ & $\begin{array}{l}2 \\
(1.1)\end{array}$ & \begin{tabular}{|l|}
116 \\
$(17.7)$ \\
\end{tabular} & $\begin{array}{l}26 \\
(14.2)\end{array}$ & $\begin{array}{l}31 \\
(17.2)\end{array}$ & $\begin{array}{l}24 \\
(14.7)\end{array}$ & $\begin{array}{l}23 \\
(20.9)\end{array}$ & $\begin{array}{l}12 \\
(25.5)\end{array}$ & $\begin{array}{l}73 \\
(21.4)\end{array}$ & \begin{tabular}{|l}
14 \\
$(19.1)$ \\
\end{tabular} & $\begin{array}{l}21 \\
(28.7)\end{array}$ & $\begin{array}{l}17 \\
(23.2) \\
\end{array}$ & \begin{tabular}{|l}
13 \\
$(17.8)$ \\
\end{tabular} & $\begin{array}{l}8 \\
(10.9) \\
\end{array}$ \\
\hline Headache & $\begin{array}{l}355 \\
(24.7)\end{array}$ & $\begin{array}{l}84 \\
(24.9)\end{array}$ & $\begin{array}{l}105 \\
(28.6)\end{array}$ & $\begin{array}{l}80 \\
(26.5)\end{array}$ & $\begin{array}{l}60 \\
(25) \\
\end{array}$ & $\begin{array}{l}26 \\
(13.7)\end{array}$ & $\begin{array}{l}313 \\
(47.7) \\
\end{array}$ & $\begin{array}{l}89 \\
(48.6)\end{array}$ & $\begin{array}{l}77 \\
(42.8)\end{array}$ & $\begin{array}{l}56 \\
(34.4)\end{array}$ & $\begin{array}{l}63 \\
(57.3)\end{array}$ & $\begin{array}{l}28 \\
(59.6)\end{array}$ & $\begin{array}{l}17 \\
(5)\end{array}$ & $\begin{array}{l}5 \\
(29.4) \\
\end{array}$ & & $\begin{array}{l}7 \\
(41.1) \\
\end{array}$ & $\begin{array}{l}4 \\
(23.5)\end{array}$ & $\begin{array}{l}1 \\
(5.8)\end{array}$ \\
\hline Dysphagia & $\begin{array}{l}32 \\
(2.2) \\
\end{array}$ & $\begin{array}{l}15 \\
(4.4) \\
\end{array}$ & $\begin{array}{l}9 \\
(2.5) \\
\end{array}$ & $\begin{array}{l}2 \\
(0.7)\end{array}$ & $\begin{array}{l}2 \\
(0.8) \\
\end{array}$ & $\begin{array}{l}4 \\
(2.1) \\
\end{array}$ & $\begin{array}{l}45 \\
(6.9) \\
\end{array}$ & $\begin{array}{l}12 \\
(6.6) \\
\end{array}$ & $\begin{array}{l}7 \\
(3.9) \\
\end{array}$ & $\begin{array}{l}9 \\
(6.6) \\
\end{array}$ & $\begin{array}{l}11 \\
(10) \\
\end{array}$ & $\begin{array}{l}6 \\
(12.8)\end{array}$ & & & & & & \\
\hline Aphonia & $\begin{array}{l}66 \\
(4.6)\end{array}$ & $\begin{array}{l}14 \\
(4.1) \\
\end{array}$ & $\begin{array}{l}15 \\
(4.1)\end{array}$ & $\begin{array}{l}18 \\
(6) \\
\end{array}$ & $\begin{array}{l}15 \\
(6.3) \\
\end{array}$ & $\begin{array}{l}4 \\
(2.1) \\
\end{array}$ & $\begin{array}{l}110 \\
(16.8)\end{array}$ & $\begin{array}{l}29 \\
(15.8)\end{array}$ & $\begin{array}{l}27 \\
(15) \\
\end{array}$ & $\begin{array}{l}22 \\
(16.2)\end{array}$ & $\begin{array}{l}23 \\
(20.9)\end{array}$ & $\begin{array}{l}9 \\
(19.1)\end{array}$ & & & & & & \\
\hline Taste loss & $\begin{array}{l}284 \\
(19.7)\end{array}$ & $\begin{array}{l}72 \\
(21.3)\end{array}$ & $\begin{array}{l}68 \\
(18.5)\end{array}$ & $\begin{array}{l}70 \\
(23.2)\end{array}$ & $\begin{array}{l}54 \\
(22.5)\end{array}$ & $\begin{array}{l}20 \\
(10.5)\end{array}$ & $\begin{array}{l}219 \\
(33.4)\end{array}$ & $\begin{array}{l}60 \\
(32.8)\end{array}$ & $\begin{array}{l}64 \\
(35.6)\end{array}$ & $\begin{array}{l}37 \\
(27.2)\end{array}$ & $\begin{array}{l}40 \\
(36.4)\end{array}$ & $\begin{array}{l}18 \\
(38.3)\end{array}$ & $\begin{array}{l}52 \\
(15.3)\end{array}$ & $\begin{array}{l}21 \\
(40.3)\end{array}$ & $\begin{array}{l}8 \\
(15.3)\end{array}$ & $\begin{array}{l}17 \\
(32.6)\end{array}$ & $\begin{array}{l}6 \\
(11.5)\end{array}$ & \\
\hline Smell loss & $\begin{array}{l}292 \\
(20.3)\end{array}$ & $\begin{array}{l}81 \\
(24)\end{array}$ & $\begin{array}{l}69 \\
(18.8)\end{array}$ & $\begin{array}{l}70 \\
(23.2)\end{array}$ & $\begin{array}{l}50 \\
(20.8)\end{array}$ & $\begin{array}{l}22 \\
(11.6)\end{array}$ & $\begin{array}{l}362 \\
(55.2)\end{array}$ & $\begin{array}{l}102 \\
(55.7)\end{array}$ & $\begin{array}{l}101 \\
(56.1)\end{array}$ & $\begin{array}{l}55 \\
(40.4)\end{array}$ & $\begin{array}{l}74 \\
(67.3)\end{array}$ & $\begin{array}{l}30 \\
(63.8)\end{array}$ & $\begin{array}{l}90 \\
(26.4)\end{array}$ & $\begin{array}{l}38 \\
(42.2)\end{array}$ & $\begin{array}{l}33 \\
(36.6)\end{array}$ & $\begin{array}{l}10 \\
(11.1)\end{array}$ & $\begin{array}{l}4 \\
(4.4)\end{array}$ & $\begin{array}{l}5 \\
(5.5)\end{array}$ \\
\hline Sore throat & $\begin{array}{c}102 \\
(7) \\
\end{array}$ & $\begin{array}{l}29 \\
(8.6) \\
\end{array}$ & $\begin{array}{l}31 \\
(8.4) \\
\end{array}$ & $\begin{array}{l}15 \\
(5) \\
\end{array}$ & $\begin{array}{l}16 \\
(6.7) \\
\end{array}$ & $\begin{array}{l}11 \\
(5.8) \\
\end{array}$ & $\begin{array}{l}238 \\
(36.3) \\
\end{array}$ & $\begin{array}{l}65 \\
(35.5) \\
\end{array}$ & $\begin{array}{l}68 \\
(37.8) \\
\end{array}$ & $\begin{array}{l}36 \\
(26.5) \\
\end{array}$ & $\begin{array}{l}50 \\
(45.5) \\
\end{array}$ & $\begin{array}{l}19 \\
(40.4) \\
\end{array}$ & & & & & & \\
\hline $\begin{array}{l}\text { Nasal } \\
\text { congestion }\end{array}$ & $\begin{array}{l}31 \\
(2.15)\end{array}$ & $\begin{array}{l}10 \\
(3)\end{array}$ & $\begin{array}{l}11 \\
(3)\end{array}$ & $\begin{array}{l}4 \\
(1.3)\end{array}$ & $\begin{array}{l}4 \\
(1.7)\end{array}$ & $\begin{array}{l}2 \\
(1.1)\end{array}$ & $\begin{array}{l}4 \\
(0.6)\end{array}$ & $\begin{array}{l}0 \\
(0)\end{array}$ & $\begin{array}{l}1 \\
(0.6)\end{array}$ & $\begin{array}{l}1 \\
(0.7)\end{array}$ & $\begin{array}{l}0 \\
(0) \\
\end{array}$ & $\begin{array}{l}2 \\
(4.3)\end{array}$ & & & & & & \\
\hline Otalgia & $\begin{array}{c}20 \\
(1.4)\end{array}$ & $\begin{array}{l}7 \\
(2.1)\end{array}$ & $\begin{array}{l}5 \\
(1.4)\end{array}$ & $\begin{array}{l}5 \\
(1.7)\end{array}$ & $\begin{array}{l}4 \\
(1.7)\end{array}$ & $\begin{array}{l}2 \\
(1.1)\end{array}$ & $\begin{array}{l}51 \\
(7.8)\end{array}$ & $\begin{array}{l}11 \\
(6)\end{array}$ & $\begin{array}{l}12 \\
(6.7)\end{array}$ & $\begin{array}{l}7 \\
(5.1)\end{array}$ & $\begin{array}{l}16 \\
(14.5)\end{array}$ & $\begin{array}{l}5 \\
(10.6)\end{array}$ & & & & & & \\
\hline $\begin{array}{l}\text { Feeling of } \\
\text { pressure in } \\
\text { the ear }\end{array}$ & $\begin{array}{l}16 \\
(1.1)\end{array}$ & $\begin{array}{l}7 \\
(2.1)\end{array}$ & $\begin{array}{l}3 \\
(0.8)\end{array}$ & $\begin{array}{l}2 \\
(0.7)\end{array}$ & $\begin{array}{l}2 \\
(0.8)\end{array}$ & $\begin{array}{l}2 \\
(1.1)\end{array}$ & $\begin{array}{l}37 \\
(5.6)\end{array}$ & $\begin{array}{l}7 \\
(3.8)\end{array}$ & $\begin{array}{l}9 \\
(5)\end{array}$ & $\begin{array}{l}3 \\
(2.2)\end{array}$ & $\begin{array}{l}10 \\
(9.1)\end{array}$ & $\begin{array}{l}8 \\
(17)\end{array}$ & & & & & & \\
\hline $\begin{array}{l}\text { Dizziness/ } \\
\text { Vertigo }\end{array}$ & $\begin{array}{l}49 \\
(3.4)\end{array}$ & $\begin{array}{l}12 \\
(3.6)\end{array}$ & $\begin{array}{l}8 \\
(2.2)\end{array}$ & $\begin{array}{l}14 \\
(4.6)\end{array}$ & $\begin{array}{l}9 \\
(3.8)\end{array}$ & $\begin{array}{l}6 \\
(3.2)\end{array}$ & $\begin{array}{l}127 \\
(19.4)\end{array}$ & $\begin{array}{l}24 \\
(13.1)\end{array}$ & $\begin{array}{l}41 \\
(22.8)\end{array}$ & $\begin{array}{l}22 \\
(16.2)\end{array}$ & $\begin{array}{l}23 \\
(20.9)\end{array}$ & $\begin{array}{l}17 \\
(36.2)\end{array}$ & $\begin{array}{l}14 \\
(4.11)\end{array}$ & $\begin{array}{l}4 \\
(28.5)\end{array}$ & & $\begin{array}{l}1 \\
(7.14)\end{array}$ & $\begin{array}{l}9 \\
(64.2)\end{array}$ & \\
\hline Tinnitus & $\begin{array}{l}14 \\
(0.9)\end{array}$ & $\begin{array}{l}5 \\
(1.5)\end{array}$ & $\begin{array}{l}5 \\
(1.4)\end{array}$ & $\begin{array}{l}2 \\
(0.7)\end{array}$ & $\begin{array}{l}2 \\
(0.8)\end{array}$ & $\begin{array}{l}0 \\
(0)\end{array}$ & $\begin{array}{l}129 \\
(19.7)\end{array}$ & $\begin{array}{l}32 \\
(17.5)\end{array}$ & $\begin{array}{l}42 \\
(23.3)\end{array}$ & $\begin{array}{l}22 \\
(16.2)\end{array}$ & $\begin{array}{l}25 \\
(22.7)\end{array}$ & $\begin{array}{l}8 \\
(17)\end{array}$ & & & & & & \\
\hline $\begin{array}{l}\text { Difficulty } \\
\text { understand } \\
\text { ing in noise }\end{array}$ & $\begin{array}{l}2 \\
(0.13)\end{array}$ & $\begin{array}{l}0 \\
(0)\end{array}$ & $\begin{array}{l}0 \\
(0)\end{array}$ & $\begin{array}{l}2 \\
(0.7)\end{array}$ & $\begin{array}{l}0 \\
(0)\end{array}$ & $\begin{array}{l}0 \\
(0)\end{array}$ & $\begin{array}{l}173 \\
(26.4)\end{array}$ & $\begin{array}{l}48 \\
(26.2)\end{array}$ & $\begin{array}{l}44 \\
(24.4)\end{array}$ & $\begin{array}{l}30 \\
(22.1)\end{array}$ & $\begin{array}{l}32 \\
(29.1)\end{array}$ & $\begin{array}{l}19 \\
(40.4)\end{array}$ & & & & & & \\
\hline $\begin{array}{l}\text { Decreased } \\
\text { sound } \\
\text { tolerance }\end{array}$ & $\begin{array}{l}2 \\
(0.13)\end{array}$ & $\begin{array}{l}0 \\
(0)\end{array}$ & $\begin{array}{l}0 \\
(0)\end{array}$ & $\begin{array}{l}2 \\
(0.7)\end{array}$ & $\begin{array}{l}0 \\
(0)\end{array}$ & $\begin{array}{l}0 \\
(0)\end{array}$ & $\begin{array}{l}42 \\
(6.4)\end{array}$ & $\begin{array}{l}10 \\
(5.5)\end{array}$ & $\begin{array}{l}10 \\
(5.6)\end{array}$ & $\begin{array}{l}8 \\
(5.9)\end{array}$ & $\begin{array}{l}6 \\
(5.5)\end{array}$ & $\begin{array}{l}8 \\
(17)\end{array}$ & & & & & & \\
\hline $\begin{array}{l}\text { Speech } \\
\text { disorder }\end{array}$ & $\begin{array}{l}2 \\
(0.13) \\
\end{array}$ & $\begin{array}{l}0 \\
(0)\end{array}$ & $\begin{array}{l}0 \\
(0)\end{array}$ & $\begin{array}{l}2 \\
(0.7)\end{array}$ & $\begin{array}{l}0 \\
(0)\end{array}$ & $\begin{array}{l}0 \\
(0)\end{array}$ & $\begin{array}{l}33 \\
(5) \\
\end{array}$ & $\begin{array}{l}6 \\
(3.3) \\
\end{array}$ & $\begin{array}{l}9 \\
(5) \\
\end{array}$ & $\begin{array}{l}7 \\
(5.1) \\
\end{array}$ & $\begin{array}{l}4 \\
(3.6) \\
\end{array}$ & $\begin{array}{l}7 \\
(14.9) \\
\end{array}$ & & & & & & \\
\hline $\begin{array}{l}\text { Nausea } \\
\text { and } \\
\text { vomiting }\end{array}$ & $\begin{array}{l}14 \\
(0.97)\end{array}$ & $\begin{array}{l}8 \\
(2.4)\end{array}$ & $\begin{array}{l}2 \\
(0.5)\end{array}$ & $\begin{array}{l}2 \\
(0.7)\end{array}$ & $\begin{array}{l}0 \\
(0)\end{array}$ & $\begin{array}{l}2 \\
(1.1)\end{array}$ & $\begin{array}{l}87 \\
(13.3)\end{array}$ & $\begin{array}{l}24 \\
(13.1)\end{array}$ & $\begin{array}{l}24 \\
(13.3)\end{array}$ & $\begin{array}{l}18 \\
(13.2)\end{array}$ & $\begin{array}{l}10 \\
(9.1)\end{array}$ & $\begin{array}{l}11 \\
(23.4)\end{array}$ & & & & & & \\
\hline
\end{tabular}

Table 3. Distribution of gender according to otologic and audio-vestibular symptoms in COVID-19

\begin{tabular}{|l|c|c|c|c|c|c|}
\hline & \multicolumn{2}{|c|}{ The 2nd days of the disease } & \multicolumn{3}{c|}{ The during 14 days of the disease } \\
\hline $\mathrm{n}$ & Male & Female & Total & Male & Female & Total \\
\hline Taste loss & 138 & 146 & 284 & 98 & 121 & 219 \\
\hline Smell loss & 150 & 142 & 292 & 141 & 221 & 362 \\
\hline Sore throat & 47 & 55 & 102 & 117 & 121 & 238 \\
\hline Nasal congestion & 17 & 14 & 31 & 1 & 3 & 4 \\
\hline Otalgia & 9 & 11 & 20 & 26 & 25 & 51 \\
\hline Feeling of pressure in the ear & 10 & 6 & 16 & 17 & 20 & 37 \\
\hline Dizziness/Vertigo & 21 & 28 & 49 & 63 & 61 & 127 \\
\hline Tinnitus & 5 & 9 & 14 & 70 & 57 & 129 \\
\hline Difficulty understanding in noise & 0 & 2 & 2 & 88 & 85 & 173 \\
\hline Decreased sound tolerance & 0 & 2 & 2 & 21 & 21 & 42 \\
\hline Speech disorder & 0 & 2 & 2 & 17 & 16 & 42 \\
\hline Nausea and vomiting & 6 & 8 & 14 & 48 & 39 & 37 \\
\hline
\end{tabular}




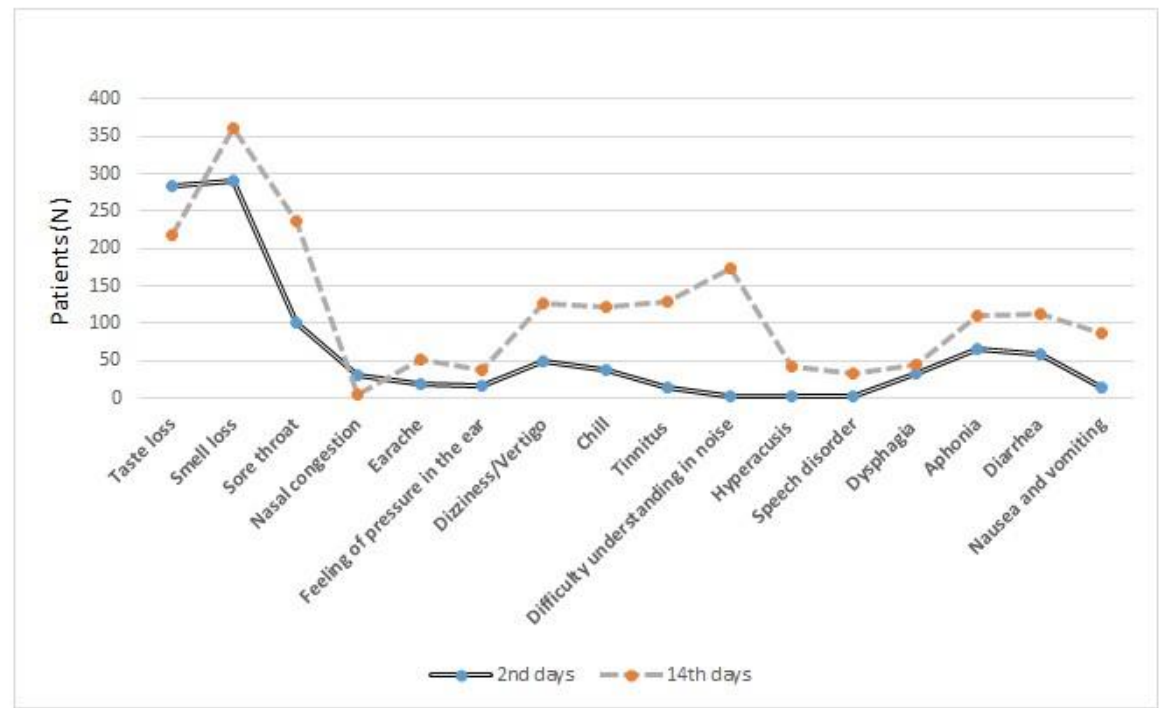

Figure 1. The percentages of patients with otologic and audio-vestibular symptoms on the second days and during 14 days of the disease.

The dashed line shows the frequency of otologic and audio-vestibular symptoms of 1437 patients on the second day of the disease, while the flat line shows the frequency of symptoms seen in 656 patients during 14 days of the disease. Percent rates of patients are given in the graph.

After three months, 340 of 1437 patients were interviewed by phone and were asked whether there were ongoing symptoms after the quarantine period. A total of $52(15.3 \%)$ patients had a loss of taste, 90 $(26.4 \%)$ patients had a loss of smell, and $17(5 \%)$ patients had frequent continued headaches. Further, $14(4.11 \%)$ of patients reported that dizziness/vertigo continued for a few weeks and then stopped. Overall, $73(21.4 \%)$ of the patients had dyspnea, especially during fast movements (Table 2).

\section{DISCUSSION}

The COVID-19 was a pandemic spread very quickly after its appearance on December 8, 2019. Because this pandemic was new, information on the symptoms caused by this disease was limited in the literature. Knowing the clinical features (symptoms) and the rate of occurrence of the disease among individuals will help create clinical admissions and treatment protocols. Our study is an expanded descriptive study on the clinical features of COVID19, especially the audio-vestibular symptoms. Additionally, we obtained useful information about the symptoms of the pandemic over time.

The coronavirus that affects humans is one of the most important of the six pathogens of respiratory tract infection (SARS-CoV, MERS-CoV, HCoVOC43, HCoV-229E, HCoV-NL63, and HCoVHKU1). Among these pathogens, SARS-CoV and MERS-CoV infection cause severe respiratory syndrome, whereas the others cause mild respiratory syndrome 21, 22. SARS-CoV (2019-nCoV) differs relatively from the other six coronaviruses ${ }^{23}$. Given that patients who start the disease with mild weakness that thereafter progress into flu-like symptoms typically continue their daily routines, if COVID-19 is ignored as a simple flu, it may play a role in the spread of the disease in public.

In our study, some patients (14.5\% of all patients) with the SARS-CoV infection had cardiovascular, anemia, hypertension, asthma, cardiovascular diseases, hypothyroid, and other comorbid diseases. Most of these patients experienced many of the symptoms of COVID-19. In a study by Chen et al. ${ }^{23-}$ 25 , about half of the patients with $2019-\mathrm{nCoV}$ infection had cardiovascular and cerebrovascular diseases and diabetes, and although this result is similar to those obtained about the MERS-CoV infection, the symptoms seen in SARS-CoV-2 and COVID-19 systematic and respiratory disorders are similar. Most of the patients participating in our study consisted of patients who were quarantined at home; 
therefore, individuals with comorbid diseases were referred to hospitals in the district and their treatment continued in the hospital, and such patients were not questioned. One of the most important observations in our study is that the symptoms seen on the second day of the disease in patients with COVID-19 differed from the symptoms seen on the later days of the disease. Although some patients stated that other symptoms increased with fatigue on the 14th day of the disease, they indicated that their symptoms worsened, especially after the 4th and 5th days of the disease. In a study conducted by Lauer et al. ${ }^{26}$, the authors noted that clinical symptoms began to appear within 14 days after exposure to the COVID-19 pandemic. In the majority of cases $(97.5 \%)$, these symptoms were observed within an average of 11.5 days after exposure (5 days after exposure to the virus). Although there is no information about when the virus was contracted in our study, most of the symptoms in the patients were observed on the 4 th and 5 th days, on average.

Although the symptoms caused by the disease were generally stated as fever, dyspnea, cough, sore throat, headache, nausea/vomiting, muscle pain, diarrhea, dyspnea, and loss of sense of smell and taste were reported. In other studies on COVID-19 3, 7, 8, 27, the Otolaryngologic (ENT) caused by the disease in our study, detailed information and percentages on audiology and general symptoms were obtained. In the first part of the study, the most common symptoms on the second day of the disease were cough, anosmia, headache, arthralgia, and notalgia, ageusia, and fatigue, although other symptoms were observed in fewer individuals. However, it was found that in the individuals who constituted the second part of our study, there was an increase in the symptoms and their rates after the second day of the disease. Especially during these periods, there was an increase in otological and audiological symptoms. In a study conducted on patients with COVID-19, it was stated that the most common symptoms were fever, cough, sore throat, headache, myalgia, and anosmia. However, these issues were not explained because the duration and time of symptoms vary. We surmise that the symptoms of cough, anosmia, headache, arthralgia, and notalgia, ageusia, and fatigue were most common in the first two days of the disease. However, the symptoms observed in the later days of the disease, especially in ENT and other audiological symptoms (dizziness or vertigo, shortness of breath, chill, tinnitus, headache, difficulty understanding in noise, being distracted by noise, speech disorder, dysphagia, dysphonia, ageusia, anosmia, sore throat, otalgia, feeling of pressure in the ear, reduced sensitivity to sound and light, diarrhea, and fever) revealed another dimension of the disease. Our study is valuable in that there has been no general and comprehensive study on these symptoms of the disease in previous studies. The prevalence of dysphonia among these patients was found to be consistent with the study on 1,081 COVID-19 positive patients conducted by Guan et $\mathrm{al}^{8}$. Thus, attention should be paid to the diagnosis and treatment of the disease in ENT and audiology clinics.

Although some viral infections are associated with auditory and vestibular functions, such as hearing loss and vertigo ${ }^{28}$, it is still unknown whether COVID-19 impairs these functions ${ }^{29}$. COVID-19 patients often suffer from smell and taste disorders, which may indicate that SARS-CoV-2 has a direct neuropathic effect $^{29}$. COVID-19 infection may lead to labyrinthitis or vestibular neuritis (as a result of the effect on the neural pathways of taste and smell), post-viral hearing loss, or vertigo ${ }^{30}$.

In our study, it was thought that the increase in complaints of vertigo, tinnitus or difficulty in understanding speech in noise, especially in the later days of COVID-19 disease, may be a result of this viral infection. In addition to these complaints, in four patients with tinnitus and in 11 patients experiencing dizziness, other complaints such as pressure in the middle ear, ear discharge, feeling of fullness in the ear, and difficulty understanding speech in noise were reported jointly. However, since these complaints can occur with many diseases such as ear fullness, increased ear pressure, and age-related diseases, it is not clear whether these symptoms are a direct/indirect result of COVID-19 disease. Therefore, the co-occurrence of tinnitus or vertigo symptoms alongside reported symptoms such as middle ear problems and/or weakness in some patients may be attributed to multiple factors.

To elucidate, a study reported that in a limited number of adult patients, acute otitis media was associated with vertigo and/or tinnitus; it was also mentioned that the limitation of this condition may be due to the accompanying mild vestibular disorders experienced by these patients ${ }^{31}$. Although COVID19 may cause involvement in the middle ear airway mucosa, the disease usually does not affect the temporal bone and rarely causes effusion in the mastoid and middle ear ${ }^{32}$. In our study, the rate of 
patients with both middle ear problems and vestibular or tinnitus complaints increased according to the second day data, which was similar to the other studies conducted ${ }^{31,32}$. In addition, mild vestibular disorders in patients with middle ear problems may have been overlooked and not reported because they may have had little effect on quality of life.

In our study, when findings such as an additional disease, ear fullness, pressure in the ear, weakness, or age-related hearing loss were excluded, it was determined that there was an increase in the rate of tinnitus and vertigo during the disease period compared to the second day of the disease in individuals under the age of 40 . Vertigo was observed in 21 patients and tinnitus was observed in 15 patients over 50 years of age without any additional complaints. It is thought that the increasing incidence of tinnitus or vertigo complaints in different age groups may have an effect on the audio-vestibular system of the direct effect of COVID 19 disease or the drugs used and/or the emotional anxiety they experience during the disease period.

We asked all patients to rate their general quality of life or the stress state they were in with a number between 0 (very bad) and 10 (very good). While the overall mean was 6.4 , the overall quality of life of 34 people with only tinnitus was found to be $7.6+2.07$. The lower quality of life of these individuals may be due to the increase in the emotional anxiety levels of the patients with the COVID-19 disease ${ }^{25}$. It was thought that the level of anxiety experienced by the elderly individuals participating in our study during the disease process would be higher than that experienced by the young ones. The mean quality of life score of individuals over the age of 50 during the 14-day disease period was $6.8 \pm 3.1$. In this case, it was believed that the reason for the complaints of tinnitus and/or vertigo made by the patients during the disease was the drugs used during COVID-19 treatment and/or the effect of infection on hearing and/or balance. Since our study is based on reports obtained from patients, these results are important in shedding light on a more detailed examination of patient complaints in future studies.

In our study, otological and audio-vestibular symptoms included dizziness or vertigo, difficulty understanding speech in noise, sensitivity to sound and light, loss of taste and smell, fever, and nasal congestion; individuals in the 30-60 age group also reported throat pain. Difficulty swallowing was found to be the most common symptom. In a study conducted on patients who were admitted to the otological and audio-vestibular clinic and were diagnosed with COVID-19, most of the otological and audio-vestibular symptoms were observed in patients in the middle age group, although other patients were not evaluated 9. In a study conducted on COVID-19-positive patients, it was stated that after the treatment was applied to the patients, the symptoms, such as coughing in young and middleaged patients, subsided more slowly than in the elderly ${ }^{33}$. In our study, dizziness/vertigo and sore throat increased more in middle-aged patients than in the first case. At the same time, the quality of life and anxiety levels of middle-aged and young individuals in the process of staying at home were more negatively affected than those of older individuals. It is thought that most of the symptoms seen in middleaged patients, such as difficulty understanding in vestibular and noisy environments, may be caused by factors such as the anxiety they experience while staying at home. Therefore, we speculate that most otological and audio-vestibular symptoms in COVID-19 patients may be observed in young and middle-aged individuals.

Although many patient groups often present to the hospital with respiratory or flu-like symptoms, otological symptoms, such as hearing loss, vertigo, and/or tinnitus, are also very important. However, they sometimes go unnoticed. As the extent of damage caused by COVID-19 infection to the auditory and/or vestibular system is still unknown, more specific research is warranted. Our study provides detailed information about the general, audio-vestibular, speech, swallowing, and other symptoms of patients with COVID-19 based on age differences within the second and fourteenth days after the disease diagnosis. The most common symptom observed on the second day of the disease was cough, whereas a significant increase was observed in audio-vestibular symptoms, especially in patients over 60 years of age, by the fourteenth day. Previous data and publications on all of these symptoms in COVID-19 cases have provided very limited information. One of the limitations of our study is that clinical symptoms could not be examined with laboratory findings due to limited patient care in hospitals in the period during which we received the patients. Thus, our study constitutes an important information source for healthcare professionals in all clinics dealing with COVID-19. However, in investigating the effects of the coronavirus, we could not ascertain whether these 
effects were caused by the side effects of the drugs administered for treating COVID-19 or by the effects of the disease.

Notably, $82.4 \%$ of the patients reported intense otological and audiological symptoms that developed before starting the medication and were relieved after using the medication. This observation needs to be researched further in future studies. More comprehensive studies on the daily changes in otological and audiological symptoms in patients will make significant contributions to the literature on COVID-19 pathophysiology. The absence of hearing tests or audio-vestibular evaluations in the findings obtained from the elderly individuals is a limitation of our study. Since our findings are based on patient reports, more detailed studies are required in the future to determine whether these results are due to COVID-19, the effects of the drugs used, or stress factors during the disease process.

Yazar Katkıları: Çalışma konsepti/Tasarımı: BÇ; Veri toplama: BÇ; Veri analizi ve yorumlama: $\mathrm{BC}$; Yazı taslağı: $\mathrm{BC}$; İçeriğin eleştire incelenmesi: $\mathrm{BC}$; Son onay ve sorumluluk: $\mathrm{BC}$; Teknik ve malzeme desteği: $\mathrm{BC}$; Süpervizyon: $\mathrm{BC}$; Fon sağlama (mevcut ise): yok. Etik Onay: Bu çalısma için Ankara Yıldırım Beyazıt Üniversitesi Sağlı Bilimleri Fakültesi Etik Kurulundan 09.07.2020 tarih ve 58 saylı karar ile etik onay alınmıştır.

Hakem Değerlendirmesi: Dış bağımsız.

Çıkar Çatışması: Yazarlar çıar çatıșması beyan etmemișlerdir. Finansal Destek: Yazarlar finansal destek beyan etmemislerdir. Yazarın Notu: Çankaya dosyalama ekibinde çalıșan uzman doktor Elif Çildir'e katkılarından ve desteklerinden dolayı teşekkür ediyorum. Author Contributions: Concept/Design : BÇ; Data acquisition: BÇ; Data analysis and interpretation: $\mathrm{BC}$; Drafting manuscript: $\mathrm{BC}$; Critical revision of manuscript: $\mathrm{BC}$; Final approval and accountability: $\mathrm{BC}$ Technical or material support: BÇ; Supervision: BÇ; Securing funding (if available): $\mathrm{n} / \mathrm{a}$.

Ethical Approval: For this study, ethical approval was obtained from the Ethics Committee of the Faculty of Health Sciences of Ankara Yildirim Beyazit University by decision No. 58 dated 09.07.2020 for this study.

Peer-review: Externally peer-reviewed

Conflict of Interest: Authors declared no conflict of interest. Financial Disclosure: Authors declared no financial support

Acknowledgement: I would like to thank Elif CILDIR the specialist doctor who works in the Çankaya filiation team, for her contribution and support.

\section{REFERENCES}

1. Sohrabi C, Alsafi Z, O'Neill N, Khan M, Kerwan A, Al-Jabir A et al. World Health Organization declares global emergency: A review of the 2019 novel coronavirus (COVID-19). Int J Surg. 2020;76:71-6.

2. Guo Y-R, Cao Q-D, Hong Z-S, Tan Y-Y, Chen S-D, Jin H-J et al. The origin, transmission and clinical therapies on coronavirus disease 2019 (COVID-19) outbreak-an update on the status. Mil Med Res. 2020;7:1-10.

3. Xu X, Yu C, Qu J, Zhang L, Jiang S, Huang D et al. Imaging and clinical features of patients with 2019 novel coronavirus SARS-CoV-2. Eur. J. Nucl. Med. 2020;47:1275-80.

4. WHO. Rolling updates on coronavirus disease (COVID-19). Geneva, WHO, 2020.

5. Zhou P, Yang X, Wang X, Hu B, Zhang L, Zhang W, et al. A pneumonia outbreak associated with a new coronavirus of probable bat origin Nature. 2020;579:270-3.

6. WHO. Clinical management of COVID-19. Geneva, WHO, 2020

7. Vaira LA, Salzano G, Deiana G, De Riu G. Anosmia and ageusia: common findings in COVID-19 patients. Laryngoscope. 2020;130:1787-1787.

8. Guan W, Ni Z, Hu Y, Liang W, Ou C, He J et al. China medical treatment expert group for Covid-19. Clinical characteristics of coronavirus disease 2019:1708-20.

9. Elibol E. Otolaryngological symptoms in COVID-19. Eur Arch Otorhinolaryngol. 2021;278:1233-6.

10. Mao L, Jin H, Wang M, Hu Y, Chen S, He Q et al. Neurologic manifestations of hospitalized patients with coronavirus disease 2019 in Wuhan, China. JAMA Neurol. 2020;77:683-90.

11. Sun P, Qie S, Liu Z, Ren J, Li K, Xi J. Clinical characteristics of hospitalized patients with SARS$\mathrm{CoV}-2$ infection: a single arm meta-analysis. J Med Virol. 2020;92:612-7.

12. Wang D, Hu B, Hu C, Zhu F, Liu X, Zhang J et al. Clinical characteristics of 138 hospitalized patients with 2019 novel coronavirus-infected pneumonia in Wuhan, China. JAMA. 2020;323:1061-9.

13. Çıldır B, Kılıç S, Ozkisi B, Tokgoz-Yilmaz S. The Turkish Short Version of the CSpeech, Spatial, and Qualities of Hearing Scale (SSQ) for clinical use: determining reliability and validity for people with and without hearing loss on the basis of SSQ12-A, SSQ12B, SSQ12-C. ENT Updates. 2021;11:127-33

14. Çıldır B, Tokgöz-Yilmaz S, Sennaroğlu G. Spatial hearing questionnaire: psychometric properties of Turkish version and correlations with hearing skills. Arch Acoust. 2021;46:249-58.

15. Aksoy S, Aslan F, Köse A. İşitme Engeli ÖlçeğiErişkin: uzun ve tarama formlarinin türkçe sürümünün geçerliğinin ve güvenirliğinin incelenmesi. KBB-Forum 2020;19:169-81.

16. Çınar BÇ, Şule K, Sjöstrand AP, Alpar R, Aksoy S. Vestibüler Bozukluklarda Günlük Yaşam Aktiviteleri Ölçeği Türkçe geçerlik ve güvenirlik çalışması. Türk Fizyoterapi ve Rehabilitasyon Dergisi. 2017;28:1-11.

17. Doruk C, Çelik M, Kara H, Polat B, Güldiken Y, Orhan KS. Turkish translation and validation of Chronic Otitis Media Questionnaire-12. Turk. Arch. Otorhinolaryngol. 2019;57:24-9.

18. Cohen J. Statistical Power Analysis for the Behavioral Sciences. Hillsdale, N J, Lawrence Erlbaum Associates, 1988.

19. Stephane Champely. pwr: Basic Functions for Power Analysis. R package version 1.3-0. (2020). 
20. R Core Team. R: A language and environment for statistical computing. R Foundation for Statistical Computing, Vienna, Austria. 2021.

21. Hu B, Zeng LP, Yang XL, Ge XY, Zhang W, Li B et al. Discovery of a rich gene pool of bat SARS-related coronaviruses provides new insights into the origin of SARS coronavirus. PLoS Pathog. 2017;13:e1006698.

22. Li W, Zhang C, Sui J, Kuhn JH, Moore MJ, Luo S et al. Receptor and viral determinants of SARScoronavirus adaptation to human ACE2. EMBO J. 2005;24:1634-43.

23. Chen N, Zhou M, Dong X, Qu J, Gong F, Han Y et al. Epidemiological and clinical characteristics of 99 cases of 2019 novel coronavirus pneumonia in Wuhan, China: a descriptive study. Lancet. 2020;395:507-13.

24. Badawi A, Ryoo SG. Prevalence of comorbidities in the Middle East respiratory syndrome coronavirus (MERS-CoV): a systematic review and meta-analysis. Int J Infect Dis. 2016;49:129-33.

25. Law S, Leung AW, Xu C. Severe acute respiratory syndrome (SARS) and coronavirus disease-2019 (COVID-19): From causes to preventions in Hong Kong. Int J Infect Dis. 2020;94:156-63.

26. Lauer SA, Grantz KH, Bi Q, Jones FK, Zheng Q, Meredith HR et al. The incubation period of coronavirus disease 2019 (COVID-19) from publicly reported confirmed cases: estimation and application. Ann Intern Med. 2020;172:577-82.
27. Pascarella G, Strumia A, Piliego C, Bruno F, Del Buono R, Costa F et al. COVID-19 diagnosis and management: a comprehensive review. Intern Med J. 2020;288:192-206.

28. Swain SK, Acharya S, Sahajan N. Otorhinolaryngological manifestations in COVID-19 infections: An early indicator for isolating the positive cases. Journal of the Scientific Society. 2020;47:63-8.

29. Lechien JR, Chiesa-Estomba CM, De Siati DR, Horoi M, Le Bon SD, Rodriguez A et al. Olfactory and gustatory dysfunctions as a clinical presentation of mild-to-moderate forms of the coronavirus disease (COVID-19): a multicenter European study. Eur Arch Otorhinolaryngol. 2020;277:2251-61.

30. Goddard JC, Fayad JN. Vestibular neuritis. Otolaryngol Clin North Am. 2011;44:361-5.

31. Choi JW, Han K, Nahm H, Shin JE, Kim C-H. Direction-changing positional nystagmus in acute otitis media complicated by serous labyrinthitis: new insights into positional nystagmus. Otol Neurotol. 2019;40:e393-8.

32. İslamoğlu $\mathrm{Y}$, Ayhan $\mathrm{M}$, Bercin $\mathrm{S}$, Kalem AK, Kayaaslan B, Güner R. Evaluation of middle ear and mastoid cells of COVID-19 patients. Journal of Ankara University Faculty of Medicine. 2021;74:1303.

33. Liu K, Chen Y, Lin R, Han K. Clinical features of COVID-19 in elderly patients: A comparison with young and middle-aged patients. J Infect.2020;80:e148. 